\title{
Slow light in photonic crystals
}

\begin{abstract}
Slow light with a remarkably low group velocity is a promising solution for buffering and time-domain processing of optical signals. It also offers the possibility for spatial compression of optical energy and the enhancement of linear and nonlinear optical effects. Photonic crystal devices are especially attractive for generating slow light as they are compatible with on-chip integration and room temperature operation and can offer wide-bandwidth and dispersion-free propagation. This paper reviews the background theory, recent experimental demonstrations and progress towards tunable slow-light structures based on photonic-band engineering. Practical issues related to real devices and their applications are also discussed.
\end{abstract}

\section{*TOSHIHIKO BABA ${ }^{1,2}$}

\author{
${ }^{1}$ Department of Electrical and Computer Engineering, \\ Yokohama National University, 79-5 Tokiwadai, \\ Hodogaya-ku, Yokohama 240-8501, Japan \\ Phone +81-45-339-4258, Fax +81-45-338-1157, e-mail: \\ baba@ynu.ac.jp \\ ${ }^{2}$ CREST, Japan Science and Technology Agency, 5 \\ Sanban-cho, Chiyoda-ku, Tokyo 102-0075, Japan
}

The velocity of light $c$ in vacuum is approximately $3 \times 10^{8} \mathrm{~m} / \mathrm{s}$, fast enough to make 7.5 round-the-world trips in a single second and to move a distance of 300 $\mathrm{mm}$ in $1 \mathrm{~ns}$ or $300 \mu \mathrm{m}$ in $1 \mathrm{ps}$. This ultrahigh speed is advantageous for efficient data transmission between two points whether they are separated on a global scale or on a single chip; however, it also makes control of optical signals in the time domain difficult. Slow light is a technology now being investigated as a means to overcome this problem.

In next-generation information networks, path switching of optical packets at network nodes will become very important and solutions that can perform the task with a high data rate, high throughput, and low power consumption are required. Engineers are now developing photonic routers that exploit all-optical processing in order to avoid optical-electronic conversion which brings many inefficiencies. Here, a key device is the optical buffer, a device which temporarily stores and adjusts the timing of optical packets. At present, solutions are based on mechanical variable delay lines and a combination of different delay lines with an optical switch but these approaches are not ideal due to their slow response. If the velocity of slow light can be controlled with a response speed much faster than the mechanical method, it could be a solution not only for buffering but also various types of time-domain processing such as re-timing, multiplexing and performing convolution integrals. Control over slow light could also improve the phase control in interferometric modulators and phased-array beam shapers. In addition, slow light offers the opportunity to compresses optical signals and optical energy in space, which reduces device footprint and enhances light-matter interactions. With enhanced optical gain, absorption and nonlinearities per unit length, numerous optical devices, such as lasers, amplifiers, detectors, absorption modulators, and wavelength converters, could be miniaturized.

The definition of velocity that is most meaningful in slow light applications is the group velocity $v_{\mathrm{g}}$ which describes the speed at which a pulse envelope propagates. In general, $v_{\mathrm{g}}$ is greatly reduced by a large first order dispersion arising from an optical resonance within a material or structure. Initially, slow light was generated using extremely strong material dispersion; however this paper discusses dispersion arising from engineered structures, in particular photonic crystal (PC) waveguides which offer a promising approach for the on-chip integration of slow-light devices.

Photonic crystals are multi-dimensional periodic structures with a period of the order of the optical wavelength $\lambda$. The research field became active in the late 1970s and 1980s (refs. 1-3) with the development of photonic band theory, an optical analogue to electronic band theory, that can be used to compute the dispersion characteristics of light in arbitrary PC structures. The theory predicted the existence of a photonic band gap (PBG), a frequency band of inhibited optical modes. Since the 1990s, PCs with PBGs have been explored for various device applications ${ }^{4-12}$. Presently, PC slabs, a high-index material with a two-dimensional array of air holes surrounded by air cladding, are widely employed because of their intrinsic lossless optical confinement and simple fabrication process. The PC waveguide (PCW) consists of a line defect of missing air holes in the $\mathrm{PC} \mathrm{slab}^{13-23}$. Light propagates through the defect, confined by total internal reflection in the vertical direction and Bragg reflection, due to the $\mathrm{PBG}$, in the lateral direction. It has been known since 2001 that the strong dispersion in this waveguide generates slow light in the vicinity of the photonic band edge $\mathrm{e}^{24-35}$.

When discussing a low $v_{\mathrm{g}}$ in a PCW, two important 
optical properties need to be considered: the frequency bandwidth of the effect and higher order dispersion ${ }^{36}$. A fundamental limit to the first of these is the delay-bandwidth product (DBP), which effects all approaches to slow light ${ }^{37-39}$. Although a wide bandwidth is desirable in most applications, it often comes at the price of less delay. The DBP means that the extent to which the group velocity of light is reduced must be balanced with the required bandwidth for the application in mind. Regarding the second issue, the higher order dispersion that usually occurs in simple slow-light PCWs severely distorts optical signals. This distortion can be eliminated either by combining two PCWs with opposite dispersion characteristics ${ }^{36,40-45}$, employing so-called dispersion-compensated slow light devices, or by suppressing the higher order dispersion using modified $\mathrm{PCWs}^{46-52}$ or a coupled-cavity optical waveguide (CROW) based on PC cavities or microrings $^{53-62}$ referred to as zero-dispersion slow light devices. Slow down of short optical pulses is now available by some of these approaches.

Once it is possible to generate wideband dispersion-free slow light, the next important consideration is tunability. A variable $v_{\mathrm{g}}$ and delay were first demonstrated for band edge slow light by externally controlling the material index of a PCW, although its bandwidth and tuning range were limited ${ }^{28}$. Later, tunability was also achieved using wideband dispersion-compensated slow light, and visible time shift of optical pulses was observed by engineering the device structure and material index control ${ }^{43}$.

Dynamic tuning of slow light, meaning that the tuning is performed while light is present in a device, is often advantageous as it provides additional unique functions such as frequency conversion and complete stopping of light in PC devices and microrings ${ }^{60-69}$. Note, however, that the delay of slow light and the potential to stop light altogether is limited in practice by the lifetime of light, which is dominated by intrinsic and extrinsic losses. In PCWs, extrinsic losses need to be conquered in order to construct feasible optical buffers ${ }^{23,70-73}$. In contrast to the demanding requirements for buffers, the enhancement of optical and optoelectronic effects is simpler to achieve, as a reduction in $v_{\mathrm{g}}$ has already been shown to be beneficial for the miniaturization and improvement of light-control devices ${ }^{52,74-85}$.

This review first presents a simple theoretical background of slow light for the better understanding of subsequent sections. It then focuses on the generation of useful slow light in PCW-based devices. Finally, some of the concepts already introduced are explained in detail and recent studies are reviewed.

\section{SLOW LIGHT THEORY AND DELAY BANDWIDTH PRODUCT}

The group velocity of light $v_{\mathrm{g}}$ is given by the inverse of the first order dispersion $(d k / d \omega)^{-1}$, where $k$ and $\omega$ are the wavenumber and angular frequency, respectively ${ }^{86}$. The group index $n_{\mathrm{g}} \equiv c / v_{\mathrm{g}}=c(d k / d \omega)$ is regarded as a slow down factor from the velocity in vacuum, $c$. Note that, in any material, the material index itself is neither very large nor easy to modify; it can be changed by no more than several percent by any form of external stimulation. Still, $n_{\mathrm{g}}$ is greatly enhanced in materials or structures with large first order dispersion.

A notable example of a system that exhibits strong material dispersion is electromagnetically induced transparency (EIT) in a vapour or a solid at, in most cases, cryogenic temperatures ${ }^{87-89}$. It is a phenomenon that arises from the coherent resonance of light with photo-excited atoms and gives rise to a huge $n_{\mathrm{g}}$ (the maximum value is of the order $10^{10}$ ). In most cases, however, EIT responds only to slowly varying optical signals because the frequency window for optical transparency is usually very narrow (of the order of $\mathrm{kHz}$ at the maximum $n_{\mathrm{g}}$ ). When a short optical pulse is incident on an EIT medium, it does not appear to be slowed, but instead resonant frequency components are filtered out, resulting in the smoothing and expanding (reshaping) of the optical pulse. A similar situation can be seen in optical resonators. In general, resonators exhibit strong structural dispersion at each resonant frequency. They form standing waves, which can be regarded as a sort of stopped light. As with EIT, resonators do not maintain the shape of the waveform of a short optical pulse.

Slow light (or stopped light) has been observed in various media, but to be useful it needs to be achieved over a wide bandwidth. Detailed analysis on the DBP has been reported in numerous papers ${ }^{37-39}$; a simple treatment is shown here. Let us denote the material index or the modal equivalent index as $n$. Using the relation $k=\omega /(c / n)$,

$$
n_{\mathrm{g}}=c \frac{d k}{d \omega}=\frac{d(n \omega)}{d \omega}=n+\omega \frac{d n}{d \omega} .
$$

When $n_{\mathrm{g}}>n$, the DBP and its normalized form are given by

$$
\Delta t \Delta f \cong \frac{L \Delta n}{\lambda} \quad \text { and } \quad n_{\mathrm{g}}\left(\frac{\Delta f}{f}\right) \cong \Delta n
$$

respectively, where $\Delta t$ is the delay over a propagation length of $L, \Delta f$ is the frequency bandwidth centred at a frequency of $f=\omega / 2 \pi$, and $\Delta n$ is the change of $n$ in the bandwidth. $\Delta f^{-1}$ approximately gives the time duration of one optical bit, although an accurate value depends on the modulation format. Therefore, the DBP $\Delta t \Delta f$ is a good measure for the highest buffering capacity that the slow light device potentially provides. On the other hand, the normalized form can be a more useful measure when devices having different lengths and different operating frequencies are compared. The shortest spatial length of one bit $\Delta L$ is approximated by

$$
\Delta L \cong \lambda / \Delta n
$$



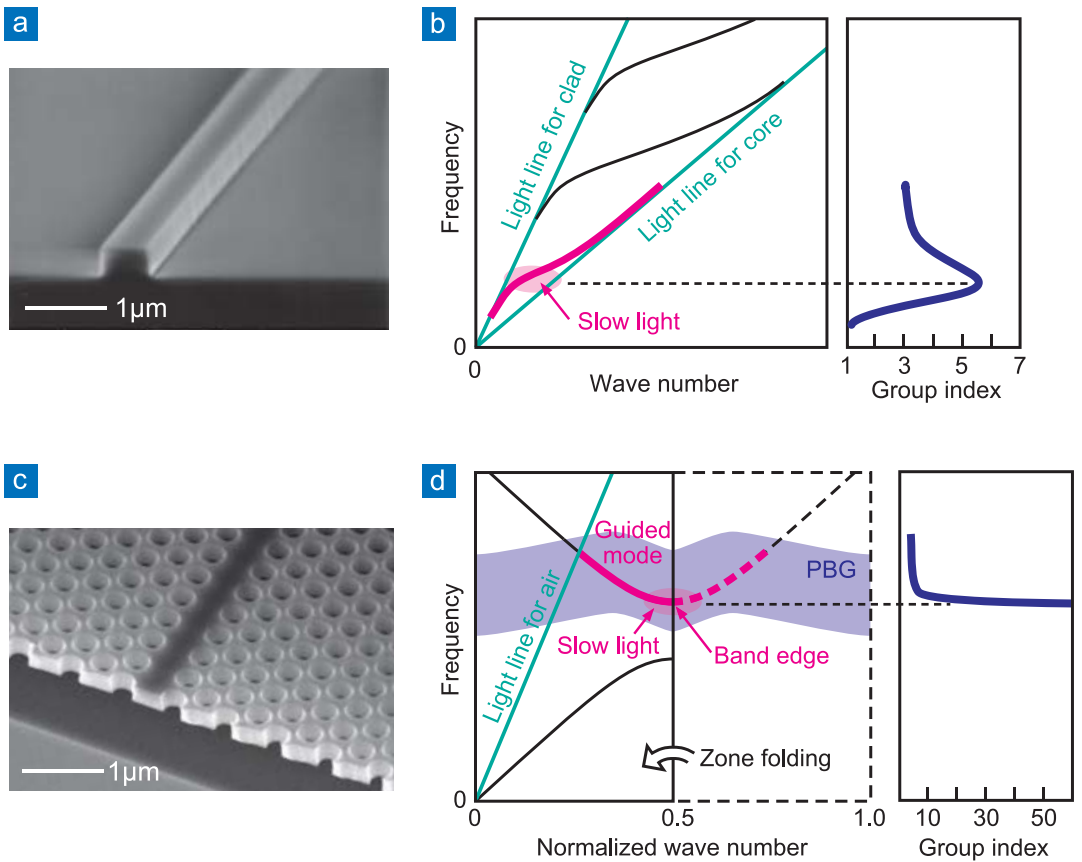
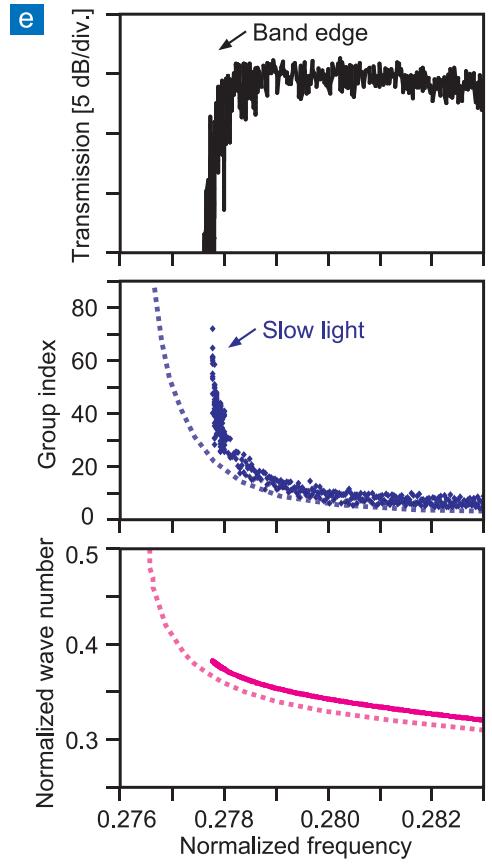

Figure 1 Waveguides, photonic bands, and group index characteristics. The normalized wave number means the wave number in units of reciprocal lattice $2 \pi / a$ where $a$ is the lattice constant. The normalized frequency is defined as $\omega a / 2 \pi c=$ $a / \lambda$. a, Scanning electron microscope (SEM) image, schematic band and group index of a silicon PWW. b, SEM image, schematic band and group index of a silicon PCW. c, Transmission spectrum, group index and band of a silicon PCW. For group index and band, dots denote experimental results obtained by the modulation phase-shift method, while dotted lines denote calculated results with an effective index approximation. Regarding C, adapted with permission from ref. 50. Copyright (2007) OSA.

Equations (2) and (3) indicate that $\Delta n$ is the dominant factor for achieving a large buffering capacity. In materials and structures with low dispersion, $\Delta n$ changes linearly with $\Delta f$ when $\Delta f<<f$. On the other hand, $\Delta n$ can be maximized in highly dispersive media independently of $\Delta f$ by optimizing photo-excitation, structural design and so on, and, according to Eq. (2), a large $n_{\mathrm{g}}$ and long $\Delta t$ are obtained by narrowing $\Delta f$. The maximum value of $\Delta n$ is usually smaller than 1.0. Taking, for example, a case where $\Delta n=0.1$ and $\Delta f=40$ $\mathrm{GHz}$ (or $40 \mathrm{kHz}$ ) at $\lambda=1.55 \mu \mathrm{m}$, then $n_{\mathrm{g}}=485$ (or $\left.4.85 \times 10^{8}\right), \Delta t=1.6 \mu \mathrm{s}(1.6 \mathrm{~s}), \Delta t \Delta f=64 \mathrm{k}(64 \mathrm{k})$ and $\Delta L$ $=15.5 \mu \mathrm{m}$ are expected for $L=1 \mathrm{~m}$. This shows the feasibility of constructing a slow-light-based optical buffer for a small number of packets, although such a long $L$ is not ideal for on-chip integration.

\section{SLOW LIGHT IN HIGHLY DISPERSIVE STRUCTURES}

For the on-chip integration and room temperature operation of slow-light devices, highly dispersive structures are more advantageous than dispersive materials. To my knowledge, the device with which slow light was first observed in 2001 was a silicon photonic wire waveguide $(\mathrm{PWW})^{90}$, which is widely used in silicon photonics devices ${ }^{91,92}$. It is a simple rectangular channel waveguide with a high index contrast (HIC) between the silicon core and air or $\mathrm{SiO}_{2}$ cladding (Fig. 1a). The propagation loss of this waveguide is sometimes measured from the finesse of the internal Fabry-Pérot resonance. In the first observation, the group index $n_{\mathrm{g}}$ was evaluated from the relation $n_{\mathrm{g}}=\lambda^{2} / 2 L \Delta \lambda_{\mathrm{r}}\left(\Delta \lambda_{\mathrm{r}}\right.$, is the peak spacing of the resonances) as $4-5$. This was not caused by the resonance but by the large dispersion arising from the HIC which largely changes the propagation constant $(k$ in the propagation direction) with $\omega$, particularly near the cutoff of the waveguide mode ${ }^{90,93}$. This result suggests that the dispersion term in Eq. (1) can be comparable to or larger than $n$ itself even in a simple waveguide. After this experiment, much larger dispersion was reported with a $\mathrm{PCW}^{25}$. Due to zone-folding of the guided-mode band and the coupling of forward and backward propagating waves forming a standing wave, the first order dispersion diverges to infinity and slow light (or stopped light) occurs near (or at) a cutoff point called the band edge (Fig. 1b). Note that similar divergence occurs in any Bragg structure; however, $\Delta n$ is typically smaller than 0.01 in shallow gratings and low index-contrast multilayer stacks. Owing to the HIC of the PC slab, the band is strongly 
deformed near the band edge and a large $\Delta n$ ranging from $0.1-1$ is obtained.

A PCW is usually fabricated on a silicon-oninsulator (SOI) or III-V compound-semiconductor substrate by using standard semiconductor processes including high-resolution lithography, selective dry etching and wet etching. A typical structure consists of air holes with a diameter $2 r$ of $240 \mathrm{~nm}$ and a lattice constant $a$ of $450 \mathrm{~nm}$ for a target wavelength $\lambda=1.55$ $\mu \mathrm{m}$; present technology means that such structures, etched to a depth of $\sim 200 \mathrm{~nm}$, can be achieved with a disorder within several nanometres. To evaluate the group velocity of the slow light, the following three methods are used: 1) frequency-domain interferometric method: measuring the spacing of the Fabry-Pérot resonances $^{90,25}$ or Mach-Zehnder interference (MZI) peaks $^{29}$, 2) time-domain modulation phase shift method: detecting the phase of light sinusoidally modulated at $\mathrm{GHz}$ frequencies ${ }^{20,42-45,49,50,52}$, and 3) time-domain direct observation of the short optical pulse transmission ${ }^{26,27,30,31,44,45,50,52}$. A rapid increase in $n_{\mathrm{g}}$ from less than ten to several tens or several hundreds is observed near the band edge using the first two methods (Fig. 1c). For the simple PCW, the third method is not easily applied because of severe higher order dispersion. Scanning near-field optical microscopy has revealed pulse broadening by capturing snapshots of the propagating pulse whose slow-light
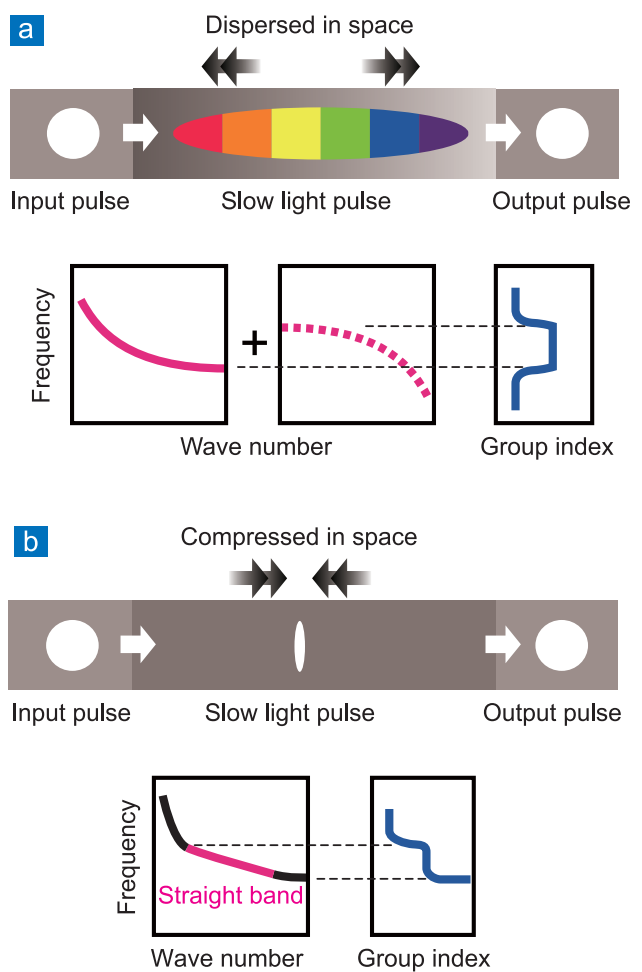

Figure 2 Schematic operation, bands and group index of dispersion-free slow-light devices. a, Dispersion-compensated slow-light device with chirped structure. b, Zero-dispersion slow-light device. part is left behind the fast parts ${ }^{30}$. The major component of the higher order dispersion is the group velocity dispersion (GVD) $d\left(v_{\mathrm{g}}{ }^{-1}\right) / d \omega=d^{2} k / d \omega^{2}$. It usually becomes extremely large near the band edge; a typical GVD constant is of the order of $100 \mathrm{ps} / \mathrm{nm} / \mathrm{mm}$, which is $10^{6}$ times larger than that of single-mode silica fibres. Because of this, dispersion-compensated slow light and zero-dispersion slow light are very important (Fig. 2). Even though a high buffering capacity is potentially expected from a large DBP in a PCW device particularly designed for wideband slow light, the net capacity is finally determined by how the GVD is suppressed.

\section{DISPERSION-COMPENSATED SLOW LIGHT}

Photonic band analysis can be used to design a device such that a positive or negative GVD in the first part of the device is cancelled out by an opposite GVD in the second part. For example, a line defect filled with air holes offset by half a period ${ }^{94}$ can create a waveguide with the opposite GVD to that of the simple PCW. These two different waveguides can be coupled together using a chirped structure ${ }^{36}$ in which some structural parameters are gradually changed along the length of the waveguides so that the guided-mode band is smoothly shifted ${ }^{28}$. Each wavelength component of light incident on the first waveguide reaches a corresponding band-edge position in the chirped structure. Band edges of these waveguides are set to always be the same in the chirped structure. Therefore, conserving $\omega$ and $k$, light is coupled to the second waveguide and simultaneously delayed near the band edges. Finally, the light propagates along and exits the second waveguide; hence, GVD of slow light is well suppressed over a wide bandwidth determined by the chirped range (completely eliminated at the centre frequency). A technical problem of this device is that a small fluctuation in the band-edge matching causes a strong oscillation in the transmission spectrum. The other option in device design is to cascade two $\mathrm{PCWs}^{41}$ where the GVD in the first part is simply compensated by the opposite GVD in the second part. In this case, the connection loss is an issue because modal profiles of these PCWs are completely different particularly near the band edge. Adiabatic tapers can reduce the loss, but then the structure usually becomes long for a compact slow-light device.

A more sophisticated device based on a similar approach but free from the matching and connection issues is the chirped PC coupled waveguide (Fig. 3a) ${ }^{42}$. It consists of two parallel PCWs whose adjacent air holes are partially enlarged or shifted to mould the band shape ${ }^{95}$. It maintains even and odd symmetric modes; the even symmetric mode shows a flat band with an inflection point that is sandwiched by the opposite GVD characteristics. This mode can be selectively used by connecting it with input/output 

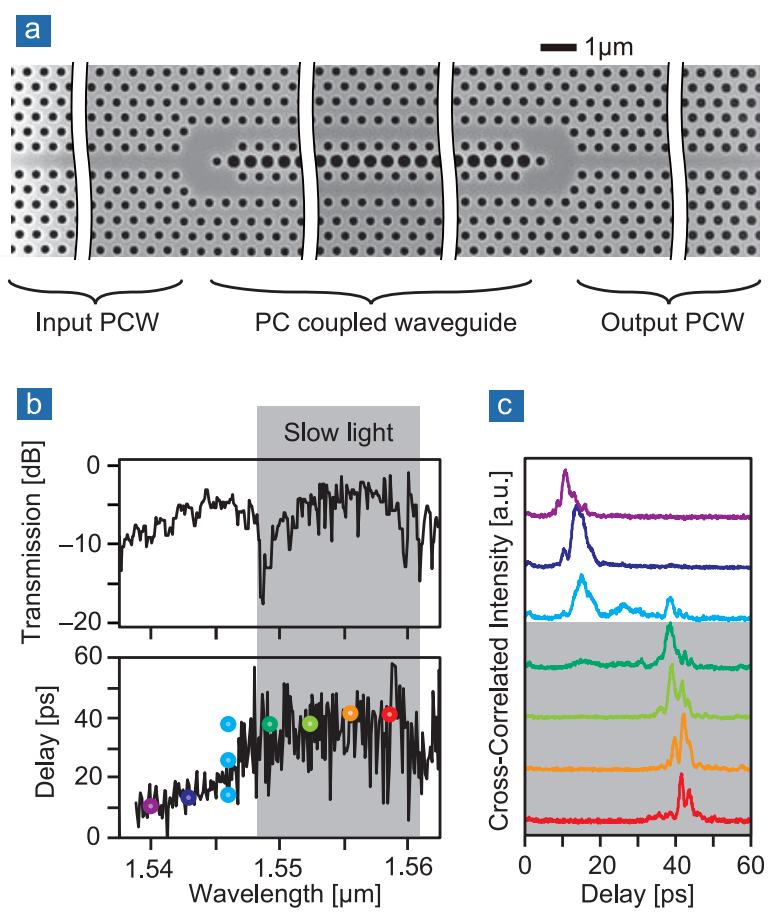

Figure 3 PC coupled waveguide and its light propagation characteristics. a, SEM image of a fabricated device on a SOI substrate. b, Measured transmission and delay spectra. Gray region shows wideband dispersion-compensated slow light. Fine oscillation is caused by the internal resonance. Circular plots correspond to the pulse delay observed in c. c, Cross-correlation trace of optical pulse at each centre wavelength denoted by colour of the circular plots in b. Adapted with permission from ref. 44 Copyright (2007) OSA, and ref. 45. Copyright (2008) OSA.

waveguides through a symmetric branch and confluence. With a chirped structure, the slow-light condition at the flat band is appropriately broadened. A simple theoretical consideration indicates that the DBP in Eq. (2) is still valid in the chirped structure by replacing $n_{g}$ with its average value. In a $L=250 \mu \mathrm{m}$ device fabricated on an SOI substrate, a $\Delta t \sim 40 \mathrm{ps}$ and $n_{\mathrm{g}}=40$ -60 were experimentally measured with a $10-12 \mathrm{~nm}$ wavelength bandwidth at $\lambda \sim 1.55 \mu \mathrm{m}(\Delta f=1.2-1.4$ $\mathrm{THz}, \Delta f / f \sim 0.7 \%)^{44,45}$. The GVD compensation in the device was confirmed from a pulse transmission experiment. Subpicosecond optical pulses were maintained at the output with some amount of dispersion even in the slow light band (Fig. 3b). The maximum DBP evaluated was 57 and corresponding $\Delta n$ was 0.35 , which approximately agrees with the expectation from Eq. (2), but the net buffering capacity was limited to 12 due to the imperfect dispersion compensation. A DBP of 1000 would be obtained by lengthening the device to $5 \mathrm{~mm}$. But to achieve a net capacity of $1 \mathrm{~kb}$, the disorder in the fabricated device must be reduced and the third and higher order dispersion $^{33,59}$ must be suppressed by further engineering a

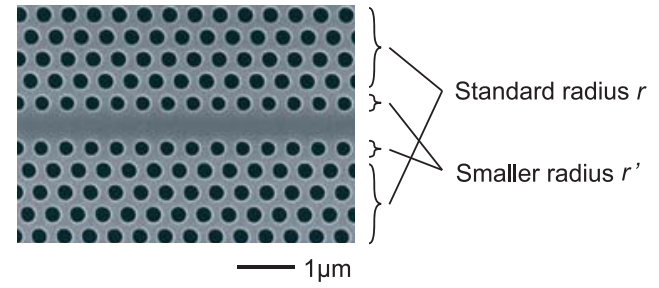

b

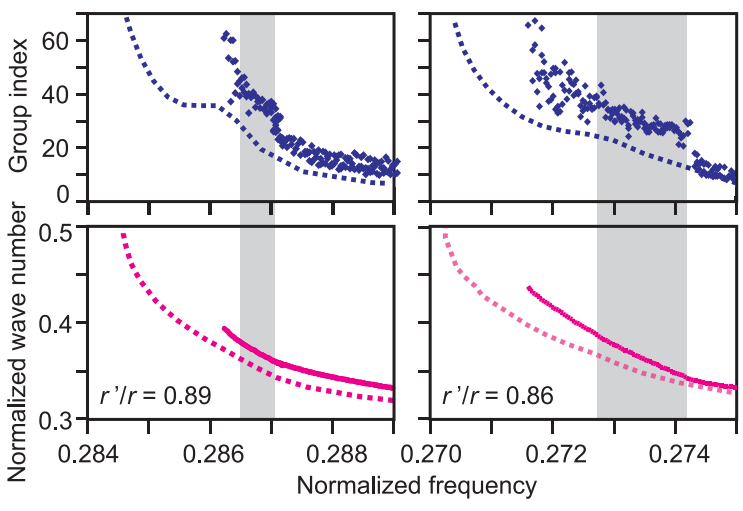

Figure 4 PCW modified for zero-dispersion slow light. a, SEM image of the top of a fabricated device on a SOI substrate. b, Group index and band structure for two ratios of air hole radius. Details are the same as those in Fig. 1c. Gray region indicating the step-like increase in group index shows the low dispersion slow light. Adapted with permission from ref. 50. Copyright (2007) OSA.

the structure and band.

In these devices, incident optical pulses are initially spatially dispersed and then recovered in the GVD compensation process. Therefore, it is effective for suppressing optical nonlinearity caused by the high intensity of the slow light.

\section{ZERO-DISPERSION SLOW LIGHT}

In fibre optics, the word zero dispersion is usually used for the zero GVD. But if the simple PCW is modified so as to give a straight guided-mode band, any higher order dispersion components are eliminated. Although the band cannot actually be completely straight, such dispersion components can be effectively reduced by this approach. In contrast to dispersion-compensated slow light, the pulse shape is compressed in space and accordingly its internal intensity is enhanced. Therefore, the approach is effective for the enhancement of optical nonlinearities.

There are a variety of ways to optimize the structure of a device to obtain a straight band. A PCW can be modified for this purpose by reducing the diameter of the innermost air holes adjacent to the line defect and increasing the diameter of the other air holes (Fig. 4a $)^{50}$. This brings the guided-mode band and 


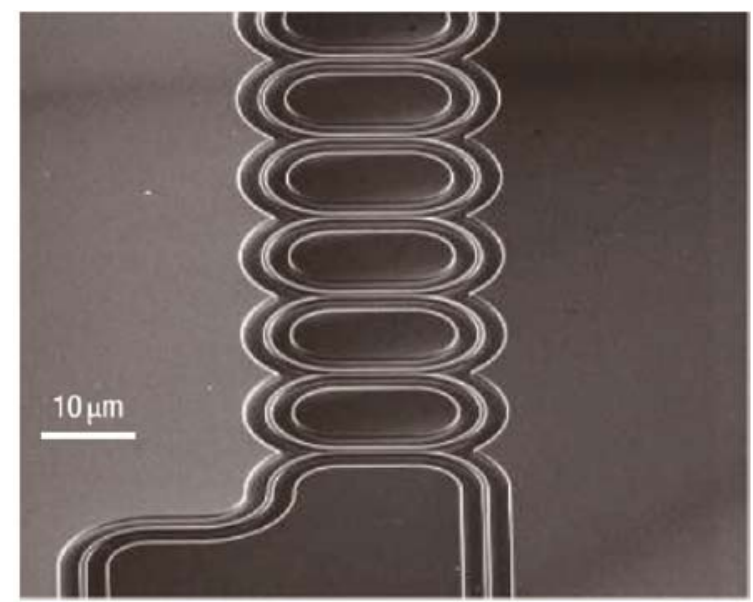

Figure 5 CROW consisting of cascaded microrings fabricated on a SOI substrate. Adapted with permission from ref. 61. Copyright (2007) Nature Photonics.

slab-mode band closer together and gives rise to their anti-crossing. When this behaviour is appropriately controlled, the guided mode band is straightened. It results in a step-like increase in $n_{\mathrm{g}}$ near the band edge and a nearly flat spectrum of $n_{\mathrm{g}}$ at the step. Fine tuning of the two air hole diameters balances $n_{\mathrm{g}}$ and the bandwidth. This type of slow light has been observed experimentally: values of $\Delta t=40-50 \mathrm{ps}$ and $n_{\mathrm{g}}=30-$ 37 were evaluated in a $11-5 \mathrm{~nm}$ wavelength bandwidth, respectively, for a $L=400 \mu \mathrm{m}$ at $\lambda \sim 1.55$ $\mu \mathrm{m}$ (Fig. 4b). The corresponding DBP is 56 and $\Delta n=$ 0.21 . The transmission of subpicosecond optical pulses was also observed as evidence of the low higher order dispersion $^{52}$.

A semi-straight band is also obtained at the centre of the transmission band of CROWs, where only the GVD is eliminated. The weak coupling between adjacent high-quality-factor microcavity resonators forms a band with a large $n_{\mathrm{g}}$ and a flat spectrum around the centre of its transmission window. Two adjacent PC nanocavities, each of which is created by shifting some of the air holes in the PC slab, exhibit two resonant peaks corresponding to coupled modes ${ }^{96}$. When $10-$ 100 of such PC microcavities are lined up to form a CROW, a transmission band appears ${ }^{62}$. As light propagates along the $\mathrm{CROW}$, localization at each cavity introduces a time delay. In this structure, the radiation loss is an intrinsic problem. Because the PC CROW has both the periodicity of the PC and the microcavities, the band is zone-folded, in addition to the original zone folding due to the PC. As a result, light is strongly diffracted, and the guided mode is easily coupled to free-radiation modes. This loss may be minimized by reducing the loss in each cavity and the coupling strength. Of course, CROWs do not necessarily involve a PC slab. Coupled microrings ${ }^{59-61}$, microdisks ${ }^{97,98}$ and microspheres ${ }^{99-101}$ have also been studied as CROWs. In a 100-cascaded-microring structure (Fig. 5), an error-free delay of 220 ps was observed for signals up to $4 \mathrm{Gbit} \mathrm{s}^{-1}$ (ref. 61). In these CROWs, a strong oscillation appears in the transmission spectrum due to disordered or even uniform coupling between cavities. Therefore the transmission of short optical pulses is still a challenge. Another type of CROW is a multi-cavity dielectric stack, which can be regarded as a one-dimensional PC with multi-periodicity ${ }^{58}$. In this structure, the radiation loss is avoided by expanding the incident optical beam, and the transmission spectrum is flattened by apodizing the layer thicknesses.

\section{TUNABILITY}

Tuning of the group index $n_{\mathrm{g}}$ is achieved by changing the material and/or modal equivalent index. To differentiate from dynamic tuning with is discussed in the next section, it is assumed here that the tuning is performed before the light enters the device.

The simplest way of tuning is to use the band-edge shift associated with a uniform index change $\Delta n$. With a small $\Delta n$, the guided-mode band of the PCW simply shifts, almost maintaining its shape, and $n_{\mathrm{g}}$ at frequencies slightly higher than the band edge change sharply. Thermal heating and carrier injection are often exploited to change the refractive index of the PC slab. Heating allows a large $\Delta n$ of up to approximately $1 \%$ and $n_{\mathrm{g}}$ was actually tuned in the range of $20-60$ using a heater integrated with a PCW on an SOI substrate ${ }^{29}$. Due to quick thermal diffusion in the small device, its response time was $100 \mathrm{~ns}$. The band-edge shift simultaneously changes the wave number $k$, leading to a large phase shift of light in the device and this observed in a MZI switch with a short device length of $160 \mu \mathrm{m}^{81}$. A much faster response is expected by using carrier plasma effects for carrier injection. The response time depends on the carrier recombination lifetime and diffusion and can be shorter than 1 ns (ref. 102), which is sufficient for optical packet buffers. In this case, $\Delta n$ has a maximum of about $0.1 \%$ resulting in a narrow tuning range.

It is important that tuning should be performed for GVD-free slow light. A change of $n_{\mathrm{g}}$ in GVD-free slow light is not obtainable by the band-edge shift approach but requires the change of the first order dispersion at the frequency of the incident light. Regarding dispersion-compensated slow light, this was achieved by engineering a sloping $\Delta n$ in addition to the structural chirping ${ }^{45}$; when the PC coupled waveguide was heated so that the temperature slope was formed, the delay of 0.9 ps wide input pulses was tuned as wide as 23 ps with an average output pulse width of $3.3 \mathrm{ps}$, and as wide as 8 ps with that of 1.2 ps (Fig. 6). For zero-dispersion slow light, the slope of the straight band must be directly changed, which may need a complicated localised change of $n$. However, an additional advantage here is that the simple shift of the 


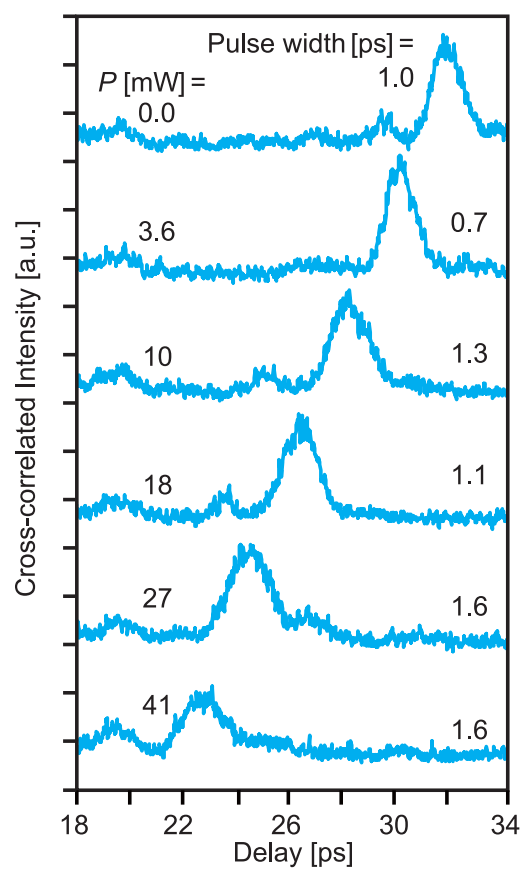

Figure 6 Delay tuning of slow light pulse in chirped PC coupled waveguide. Subpicosecond optical pulses were incident on the device. Wideband dispersion- compensated slow light is obtained by chirping the air hole diameter of the device. The delay was externally controlled by the laser heating with power $P$, which formed the slope of the temperature and corresponding material index and changed the effective amount of chirping. Adapted with permission from ref. 45. Copyright (2008) OSA.

straight band with the uniform $\Delta n$ gives a large phase change in the device, keeping the same group velocity, thus improving the performance of MZI modulators and switches.

\section{DYNAMIC TUNING FOR THE STOPPING OF LIGHT}

Thus far, slow light has been thought of in terms of an invariant frequency spectrum that does not change within the device. If wideband optical signals are converted into a very narrow spectrum, $n_{\mathrm{g}}$ can be freely enhanced, according to Eq. (2). In the extreme case that $\Delta f$ approaches zero, $n_{\mathrm{g}}$ diverges and the light is completely stopped. Strictly speaking, light does not stop; its back and forth motion forms a standing wave, storing an optical energy, and just appears to be stopped. But the DBP no longer represents a constraint for such light if the information carried by the signals is not lost during the process. The information is actually stored in the spatial distribution of light as the wave number $k$ is conserved. Such an operation is achievable by dynamic tuning, which is a type of parametric tuning process ${ }^{66}$. As observed in interferometric modulators, a temporal change of $n$ leads to a phase and frequency shift of light passing through the material. Suppose that $n$ in a waveguide is changed quickly and adiabatically within the time that optical signals are still present in the waveguide. In this situation, the wavelength (frequency) of light is changed in (inverse) proportion to $n$, while almost maintaining the spatial distribution. If the waveguide is designed so that $n_{\mathrm{g}}$ diverges with $\Delta n$, then the signal bandwidth is compressed into a single frequency by the dynamic tuning and the light is simultaneously stopped. A structure that has been theoretically discussed is a system consisting of a PCW coupled with an adjacent series of PC cavities, which can, in total, be regarded as a $\mathrm{CROW}^{64}$. In this case, the coupling strength is controlled by a local $\Delta n$ so that $n_{\mathrm{g}}$ in the system is increased. It is equivalent to the dynamic increase in the quality factor of each cavity, so light propagating in the PCW is caught in the cavities. If long and complicated optical signals are present in the device during the tuning, they are divided into pieces and the envelope distribution is transcribed into the series of cavities. A reverse process that kills $\Delta n$ faithfully recovers the initial light propagation. Experimentally such dynamic tuning has been demonstrated for a single optical pulse in a coupled system comprising of a PCW and a PC single cavity with a phase shifter ${ }^{68}$ and that comprising of a PWW and a couple of microrings ${ }^{69}$. The coupling condition was controlled using the carrier plasma effect, and the temporal catch and release of a pulse was demonstrated. Regarding the latter structure, a variable transmission spectrum similar to that in EIT was also studied ${ }^{103,104}$.

A drawback of the dynamic tuning, compared with simple slow light, is the large device footprint needed for storing a series of optical signals. The envelope distribution of the optical signals is not compressed in space during the dynamic tuning but just stopped as it is. The number of signals stored in the device is limited up to 400 for $L=1 \mathrm{~m}, n=3$ and $\Delta f=40 \mathrm{GHz}$. This constraint could be relaxed by the pre-compression of light in a slow light device before the dynamic tuning ${ }^{63-65}$. Another constraint is that once the dynamic tuning is performed, subsequent signals cannot be input to the device until the reverse process is completed. Therefore, this process is suitable for optical RAM operation.

\section{EXTRINSIC LOSS}

In a well designed PCW, the guided mode band is ideally loss-less due to the optical confinement by the PBG in the lateral direction and by the total internal reflection in the vertical direction. Actually, however, the light scattering owing to structural disorder and material absorption give rise to a loss. As estimated above, buffering on the microsecond time scale needs a device length $L=1 \mathrm{~m}$ even for slow light. The total 
loss must be within a range that can be recovered by the gain of a standard optical amplifier, e.g. $<30 \mathrm{~dB}$ in the worst case. The waveguide loss should be lower than $0.3 \mathrm{~dB} \mathrm{~cm}^{-1}$ in the slow light regime.

The reduction in scattering loss is, in some senses, the most crucial problem. The scattering loss in conventional index-confinement waveguides depends approximately on $\Delta^{2.5}$, where $\Delta \equiv\left(n_{1}{ }^{2}-n_{2}{ }^{2}\right) / 2 n_{1}{ }^{2}$ is the relative refractive index difference between the core index $n_{1}$ and cladding index $n_{2}$ (ref. 105). Therefore, the loss in HIC waveguides, such as silicon PWWs where $\Delta>40 \%$, can be $10^{4}-10^{5}$-fold higher than that of conventional silica-based waveguides where $\Delta<$ $0.5 \%$. Nevertheless, the loss in a PWW has been reduced to $\sim 1 \mathrm{~dB} \mathrm{~cm}^{-1}$ by recent technical improvements ${ }^{106-108}$. The loss in the PCW on a SOI substrate, which has the same $\Delta$, is expected to be lower because the scattering of the guided mode into radiation modes could be suppressed by the $\mathrm{PBG}^{18}$. However, losses measured so far are still higher. In addition, low loss is limited to the low- $n_{\mathrm{g}}$ regime; much larger losses are observed near the band edge. There are active discussions about the dependence of loss on $n_{\mathrm{g}}$. Some experimental results suggest $n_{\mathrm{g}}{ }^{2}$ and $\sqrt{ } n_{\mathrm{g}}$ dependences, which are attributed to doubly enhanced back-scattering and the change in modal profile respectively ${ }^{23,73}$. This must be further investigated to suppress the loss in a practical design.

The interband absorption should be negligible when the material bandgap $E_{\mathrm{g}}$ is larger than the photon energy. But the absorption extends to longer wavelengths in indirect-bandgap materials such as silicon. Also, absorption centres could be introduced into the top silicon layer of commercially available SOI wafers during fabrication. Also, nonlinear two-photon absorption (TPA) in silicon will be a problem at $\lambda \sim$ $1.55 \mu \mathrm{m}$; it scales up with $n_{\mathrm{g}}{ }^{2}$. Once TPA occurs, excited carriers lead to self-phase modulation (SPM) and free-carrier absorption (FCA). This has been observed in silicon PWWs when the waveguide is millimetre to centimetre long and the incident power reaches a watt order ${ }^{109-112}$. These effects can occur for a much lower power and/or in a much shorter waveguide with a large $n_{\mathrm{g}}$ of slow light. Actually, they were observed for ps optical pulses in a zero-dispersion slow light PCW with a $0.15 \mathrm{~W}$ power and an effective slow light length of $300 \mu \mathrm{m}^{52}$. To completely suppress TPA, a material with $E_{\mathrm{g}}$ larger than twice the photon energy at $\lambda \sim 1.55 \mu \mathrm{m}$ is desired for a PC slab ${ }^{113}$.

An additional loss associated with slow light occurs in the coupling between slow light devices and input/output devices. When these devices are connected directly, slow light with an exotic modal profile is strongly reflected and/or scattered. Some adiabatic structures are effective for the transformation of the modal profile between a PWW and a PCW ${ }^{114-117}$. Also, almost negligible reflection, less than $-30 \mathrm{~dB}$, is calculated for the chirped PC coupled waveguide, in which the incident light is gradually transformed into slow light ${ }^{42}$.

\section{ENHANCED LIGHT-MATTER INTERACTION}

In lasers, amplifiers and absorption modulators, gain and absorption coefficients per unit length increase linearly with $n_{\mathrm{g}}$ when those per unit time are constant. As mentioned above, resonators can be thought of as light stopping devices at certain frequencies. In PCs fabricated with III-V materials, laser operation occurs at band edges ${ }^{118-122}$. This is usually explained as a distributed feedback (DFB) effect, but can also be treated as a slow-light effect. Let us consider a PCW laser $^{123-125}$ perfectly open to free space, as an example. The photon lifetime in this waveguide is equivalent to the time for light propagation, and it is extended with $n_{\mathrm{g}}$, so light acquires sufficient gain for laser operation. In practice, some reflection occurs at the waveguide ends, and this determines the phase and wavelength of the resonance. Consequently, the lasing wavelengths are not precisely aligned with the band edge but are slightly shifted into the waveguide band. Just as happens in the transmission spectrum of the passive PCW, the photo-pumped lasing spectrum in a GaInAsP PCW exhibits a narrowing of the Fabry-Pérot modal spacing toward the band edge ${ }^{124}$.

The enhanced gain and absorption are expected to reduce the size of semiconductor optical amplifiers $(\mathrm{SOAs})^{83}$ and electro-absorption (EA) modulators ${ }^{85}$. A simple theoretical estimation shows a high modal gain of $25 \mathrm{~dB}$ in a 10- $\mu \mathrm{m}$-long GaInAsP SOA with $n_{\mathrm{g}}=100$ and an injection current of $3 \mathrm{~mA}$. Due to the small device size, reasonably low current level, and severe thermal heating under continuous wave condition, an output power higher than $10 \mathrm{dBm}$ would not be expected for such a small device making it suitable as a pre-amplifier and a loss compensator in photonic circuits. A short EA modulator based on slow light was demonstrated using a vertical cavity with oblique incidence of light. A big challenge that remains in regard to these devices is the integration of electrical elements with a PCW. PCWs comprising of an active layer and $\mathrm{p}$ - and n-type semiconductor claddings with perforated deep air holes have been studied to enable current injection and to apply a voltage.

Second and third-order optical nonlinearities are also enhanced as $n_{\mathrm{g}}{ }^{2}$. TPA, another propagation loss mechanism, can be used to create an optical limiter. Similarly, SPM, four-wave mixing, super-continuum generation, and Raman amplification have been observed in silicon PWWs ${ }^{109-113,126-131}$. Here, TPA and associated FCA often become a problem because the waveguide channel is electronically isolated. The zero-dispersion slow light PCW is expected to enhance not only TPA and SPM but also other nonlinearities mentioned above and decrease FCA due to fast carrier diffusion with a lifetime of less than $1 \mathrm{~ns}$ as it is electrically connected with other parts of the silicon layer. 


\section{SUMMARY}

Slow light with a group velocity several tens to several hundreds of times lower than $c$ is attainable with present PCW-based technology. This is an impressive result when considering the likely bandwidth requirements for future data traffic, above $40 \mathrm{GHz}$. The delay-bandwidth product will soon reach $100-1000$ by increasing the device length to the millimetre or centimetre scale and by reducing extrinsic losses. Dispersion management and tunability add greatly to the value of slow light. Owing to dispersioncompensated and zero-dispersion structures, slow propagation of subpicosecond optical pulses has been confirmed. Wide-range delay tuning has also been achieved for the dispersion-compensated slow light. This unique functionality was previously only available in mechanically variable delay lines, with commercial devices offering a tuning response time on the millisecond timescale. The absolute value of the delay in PC devices is limited to less than $1 \mathrm{~ns}$. The extrinsic loss, mainly due to light scattering, is the most crucial problem to be overcome and must be suppressed by further structure optimization and improvements to the fabrication process. Zero-dispersion slow light pulses enable studies on enhanced light-matter interaction. Particularly, the enhancement of optical nonlinearities has started to be observed experimentally, which is of great interest for adding complex functions in PC integrated circuits.

\section{References}

1. Ohtaka, K. Energy band of photons and low-energy photon diffraction. Phys. Rev. B 19, 5057-5067 (1979).

2. Yablonovitch, E. Inhibited spontaneous emission in solid-state physics and electronics. Phys. Rev. Lett. 58, 2059-2062 (1987).

3. John, S. Strong localization of photons in certain disordered dielectric superlattices. Phys. Rev. Lett. 58, 2486-2489 (1987).

4. Bowden, C. M., Dowling, J. P. \& Everitt, H. O. Special issue on development and applications of materials exhibiting photonic band gaps. J. Opt. Soc. Am. B 10-2, 280-413 (1993).

5. Joannopoulos, J. D., Meade, R. D. \& Winn, J. N. Photonic Crystals - Moulding the Flow of Light (Princeton University Press, Princeton, 1995).

6. Photonic Band Gap Materials (ed Soukoulis, C. M.) (Kluwer, Dordrecht, 1996).

7. Scherer, A., Doll, T., Yablonovitch, E., Everitt, H. O., \& Higgins, J. A. Special issue on electromagnetic crystal structures, design, synthesis, and applications. J. Lightwave Technol. 17, 1928 (1999).

8. Photonic Crystals and Light Localization in the $21^{\text {st }}$ Century (ed Soukoulis, C. M.) (Kluwer, Dordrecht, 2001).

9. Krauss, T. K. \& Baba, T. Feature section on photonic crystal structures and applications. IEEE J. Quantum Electron. 38, 724-956 (2002).

10. Roadmap on photonic crystals (eds Noda, S. \& Baba, T.) (Kluwer, Norwell, 2003).

11. Photonic Crystals Physics, Fabrication and Applications (eds Inoue, K. \& Ohtaka, K.) (Springer, Berline, 2004).

12. Joannopoulos, J. D., Johnson, S. G., Winn, J. N. \& Meade, R. D. Photonic Crystals - Moulding the Flow of Light $2^{\text {nd }} E d$. (Princeton University Press, Princeton, 2008).

13. Baba, T., Fukaya, N. \& Yonekura, J. Observation of light transmission in photonic crystal waveguides with bends. Electron. Lett. 35, 654-655 (1999).

14. Loncar, M., Nedeljkovic, D., Doll, T., Vuckovic, J. \& Scherer, A Waveguiding in planar photonic crystals. Appl. Phys. Lett. 77, 1937-1939 (2000).

15. Smith, C. J. M., et al. Low-loss channel waveguides with two-dimensional photonic crystal boundaries. Appl. Phys. Lett. 77, 2813-2815 (2000)

16. Noda, S., Chutinan, A. \& Imada, M. Trapping and emission of photons by a single defect in a photonic bandgap structure. Nature 407, 608-610 (2000).

17. Notomi, M., et al. Singlemode transmission within photonic bandgap of width-varied single-line-defect photonic crystal waveguides on SOI substrates. Electron. Lett. 37, 293-295 (2001).

18. Baba, T., et al. A. Light propagation characteristics of straight single line defect optical waveguides in a photonic crystal slab fabricated into a silicon-on-insulator substrate. IEEE J. Quant. Electron. 38, 743-752 (2002).

19. Sugimoto, Y., et al. Low propagation loss of $0.76 \mathrm{~dB} / \mathrm{mm}$ in GaAs-based single-line-defect two-dimensional photonic crystal slab waveguides up to $1 \mathrm{~cm}$ in length. Opt. Express 12, 1090-1096 (2004).

20. Notomi, M., et al. Waveguides, resonators and their coupled elements in photonic crystal slabs. Opt. Express 12, 1551-1561 (2004).

21. Bogaerts, W., et al. Nanophotonic waveguides in silicon-on-insulator fabricated with CMOS technology. $J$. Lightwave Technol. 23, 401-412 (2005).

22. Dulkeith, E., McNab, S. J. \& Vlasov, Y. A. Mapping the optical properties of slab-type two-dimensional photonic crystal waveguides. Phys. Rev. B 72, 115102 (2005).

23. Kuramochi, E., et al. Disorder-induced scattering loss of line-defect waveguides in photonic crystal slabs. Phys. Rev. B. 72, 161318 (2005).

24. Letartre, X., et al. Group velocity and propagation losses measurement in a single-line photonic-crystal waveguide on InP membranes. Appl. Phys. Lett. 79, 2312-2314 (2001).

25. Notomi, M., et al. Extremely large group-velocity dispersion of line-defect waveguides in photonic crystal slabs. Phys. Rev. Lett. 87, 253902 (2001)

26. Inoue, K., et al. Observation of small group velocity in two-dimensional AlGaAs-based photonic crystal slabs. Phys. Rev. Lett. B 65, 121308 (2002).

27. Asano, T., Kiyota, K., Kumamoto, D., Song, B. S. \& Noda, S. Time-domain measurement of picosecond light-pulse propagation in a two-dimensional photonic crystal-slab waveguide. Appl. Phys. Lett. 84, 4690-4692 (2004).

28. Baba, T., Mori, D., Inoshita, K. \& Kuroki, Y. Light localization in line defect photonic crystal waveguides. IEEE J. Quant. Electron. 10, 484-491 (2004).

29. Vlasov, Y. A., O’Boyle, M., Hamann, H. F. \& McNab, S. J. Active control of slow light on a chip with photonic crystal waveguides. Nature 438, 65-69 (2005).

30. Gersen, H., et al. Real-space observation of ultraslow light in photonic crystal waveguides. Phys. Rev. Lett. 94, 073903 (2005).

31. Finlayson, C. E., et al. Slow light and chromatic temporal dispersion in photonic crystal waveguides using femtosecond time of flight. Phys. Rev. Lett. 73, 016619 (2006).

32. Engelen, R. J. P., et al. The effect of higher-order dispersion on slow light propagation in photonic crystal waveguides. Opt. Express 14, 1658-1672 (2006).

33. Tanaka, Y., et al. Effect of third-order dispersion on subpicosecond pulse propagation in photonic-crystal waveguides. Appl. Phys. Lett. 89, 131101 (2006).

34. Baba, T. \& Mori, D. Slowlight engineering in photonic crystals. J. Phys. D: Appl. Phys. 40, 2659-2665 (2007).

35. Krauss, T. Slow light in photonic crystal waveguides. J. Phys. D: Appl. Phys. 40, 2666-2670 (2007).

36. Mori, D. \& Baba, T. Dispersion-controlled optical group delay device by chirped photonic crystal waveguides. Appl. Phys. Lett. 85, 1101-1103 (2004).

37. Tucker, R. S., Ku, P-C. \& Chang-Hasnain, C. J. Slow-light optical buffers - capabilities and fundamental limitations. $J$. 
Lightwave Technol. 23, 4046-4066 (2005).

38. Khurgin, J. B. Optical buffers based on slow light in electromagnetically induced transparent media and coupled resonator structures: comparative analysis. J. Opt. Soc. Am. B 22, 1062-1074 (2005).

39. Miller, D. A. B. Fundamental limit to linear one-dimensional slow light structures. Phys. Rev. Lett. 99, 203903 (2007).

40. Mori D. \& Baba, T. Wideband and low dispersion slow light by chirped photonic crystal coupled waveguide. Opt. Express 13, 9398-9408 (2005).

41. Povinelli, M. L., Johnson, S. G. \& Joannopoulos, J. D. Slow-light, band-edge waveguides for tunable time delays. Opt. Express 13, 7145-7159 (2005).

42. Mori, D., Kubo, S., Sasaki H. \& Baba, T. Experimental demonstration of wideband dispersion-compensated slow light by a chirped photonic crystal directional coupler. Opt. Express 15, 5264-5270 (2007).

43. Huang, S. C., Kato, M., Kuramochi, E., Lee, C. P. \& Notomi, M. Time-domain and spectral-domain investigation of inflection-point slow-light modes in photonic crystal coupled waveguides. Opt. Express 15, 3543-3549 (2007).

44. Kawasaki, T., Mori, D. \& Baba, T. Experimental observation of slow light in photonic crystal coupled waveguides. Opt. Express 15, 10274-10281 (2007)

45. Baba, T., Kawasaki, T., Sasaki, H., Adachi, J. \& Mori, D. Large delay-bandwidth product and delay tuning of slow light pulse in photonic crystal coupled waveguide. Opt. Express 16, 9245-9253 (2008).

46. Sakai, A., Kato, I., Mori, D. \& Baba, T. Anomalous low group velocity and low dispersion in simple photonic crystal line defect waveguides. Tech. Dig. IEEE/LEOS Annual Meet. ThQ5 (Puerto Rico, IEEE/LEOS, 2004).

47. Petrov, A. Yu. \& Eich, M. Zero dispersion at small group velocities in photonic crystal waveguides. Appl. Phys. Lett. 85, 4866-4868 (2004).

48. Settle, M. D., et al. Flatband slow light in photonic crystals featuring spatial pulse compression and terahertz bandwidth. Opt. Express 15, 219-226 (2007).

49. Frandsen, L. H., Lavrinenko, A. V., Fage-Pedersen, J. \& Borel, P. I. Photonic crystal waveguides with semislow light and tailored dispersion properties. Opt. Lett. 14, 9444-9446 (2006).

50. Kubo, S., Mori, D. \& Baba, T. Low-group-velocity and low-dispersion slow light in photonic crystal waveguides. Opt. Lett. 32, 2981-2983 (2007)

51. Li, J., White, T. P., O'Faolain, L., Gomez-Iglesias, A. \& Krauss, T. F. Systematic design of flat band slow light in photonic crystal waveguides. Opt. Express 16, 6227-6232 (2008).

52. Hamachi, Y., Kubo, S. \& Baba, T. Low dispersion slow light and nonlinearity enhancement in lattice-shifted photonic crystal waveguide. Tech. Dig. Quantum Electron. Laser Sci. Conf., QTuC1 (San Jose, OSA, 2008).

53. Yariv, A., Xu, Y., Lee, R. K. \& Scherer, A. Coupled-resonator optical waveguide - a proposal and analysis. Opt. Lett. 24, 711-713 (1999).

54. Oliver, S., et al. Miniband transmission in photonic crystal coupled resonator optical waveguide. Opt. Lett. 26, 1019-1021 (2001).

55. Hosomi, K. and Katsuyama, T. A dispersion compensator using coupled defects in a photonic crystal. IEEE J. Quantum Electron. 38, $825-829$ (2002).

56. Martinez, A., et al. Group velocity and dispersion model of coupled-cavity waveguides in photonic crystals. J. Opt. Soc. Am. A 20, 147-150 (2003).

57. Kim, W. J., Kuang, W. \& O’Brien, J. D. Dispersion characteristics of photonic crystal coupled resonator optical waveguides. Opt. Express 25, 3431-3437 (2003).

58. Fukamachi, T., Hosomi, K., Katsuyama, T. \& Arakawa, Y. Group-delay properties of coupled-defect structures in photonic crystals. Jpn. J. Appl. Phys. 43, L449-L452 (2004).

59. Khurgin, J. B. Expanding the bandwidth of slow-light photonic devices based on coupled resonators. Opt. Lett. 30, 513-515 (2005).

60. Poon, J. K., Zhu, L., De Rose, G. A. \& Yariv, A. Transmission and group delay of microring coupled-resonator optical waveguides. Opt. Lett. 31, 456-458 (2006).

61. Xia, F., Sekaric, L. \& Vlasov, Y. Ultracompact optical buffers on a silicon chip. Nature Photon. 1, 65-71 (2007).

62. Kuramochi, E., Tanabe, T., Taniyama, H., Kato, M. \& Notomi, M. Observation of heavy photon state in ultrahigh-Q photonic crystal coupled resonator chain. Tech. Dig. Quantum Phys. \& Laser Sci. Conf., QMG2 (Baltimore, OSA, 2007).

63. Yanik, M. F. \& Fan, S. Stopping light all optically. Phys. Rev. Lett. 92, 083901 (2004).

64. Yanik, M. F., Suh, W., Wang, Z. \& Fan, S. Stopping light in a waveguide with an all-optical analog of electromagnetically induced transparency. Phys. Rev. Lett. 93, 233903 (2004).

65. Khurgin, J. B. Adiabatically tunable optical delay lines and their performance limitations. Opt. Lett. 30, 2778-2780 (2005).

66. Notomi, M., Mitsugi, S. Wavelength conversion via dynamic refractive index tuning of a cavity. Phys. Rev. A 73, 051803 (2006).

67. Preble, S. F., Xu, Q. \& Lipson, M. Changing the colour of light in a silicon resonator. Nature Photon. 1, 293-296 (2007).

68. Tanaka, Y., et al. Dynamic control of the $\mathrm{Q}$ factor in a photonic crystal nanocavity. Nature Mater. 6, 862-865 (2007).

69. Xu, Q., Dong, P. \& Lipson, M. Breaking the delay-bandwidth limit in a photonic structure. Nature Phys. 3, 406-410 (2007).

70. Tanaka. Y., et al. Group velocity dependence of propagation losses in single-line-defect photonic crystal waveguides on $\mathrm{GaAs}$ membranes. Electron. Lett. 40, 174-176 (2004)

71. Hughes, S., et al. Extrinsic optical scattering loss in photonic crystal waveguides - Role of fabrication disorder and photon group velocity. Phys. Rev. Lett. 94, 033903 (2005).

72. Mookherjea, S. \& Oh, A. Effect of disorder on slow light velocity in optical slow-wave structures. Opt. Lett. 32, 289-291 (2007).

73. O'Faolain, L., et al. Dependence of extrinsic loss on group velocity in photonic crystal waveguides. Opt. Express 15, 13129-13138 (2007).

74. Soljacic, M., et al. Photonic-crystal slow-light enhancement of nonlinear phase sensitivity. J. Opt. Soc. Amer. B 19, 2052-2059 (2002).

75. Konorov, S. O., et al. Coherent anti-Stokes Raman scattering of slow light in a hollow planar photonic band-gap waveguide. Laser Phys. 12, 818-824 (2002).

76. Soljacic, M., et al. Nonlinear photonic crystal microdevices for optical integration. Opt. Lett. 28, 637-639 (2003).

77. Soljacic, M. \& Joannopoulos, J. D. Enhancement of nonlinear effects using photonic crystals. Nature Mater. 3, 211-219 (2004).

78. Nakamura, H., et al. Ultra-fast photonic crystal/quantum dot all-optical switch for future photonic networks. Opt. Express 12, 6606-6614 (2004).

79. Camargo, E. A., Chong, H. M. \& De la Rue, R. M. 2D photonic crystal thermo-optic switch based on $\mathrm{AlGaAs} / \mathrm{GaAs}$ epitaxial structure. Opt. Express 12, 588-592 (2004).

80. Raineri, F., et al. Optical amplification in two-dimensional photonic crystals. Appl. Phys. Lett. 86, 091111 (2005).

81. Chu, T., Yamada, H., Ishida, S. \& Arakawa, Y. Thermooptic switch based on photonic-crystal line-defect waveguides. IEEE Photon. Technol. Lett. 17, 2083-2085 (2005).

82. Oda, H. \& Inoue, K. Observation of Raman scattering in GaAs photonic-crystal slab waveguides. Opt. Express 14, 6659-6667 (2006).

83. Mizuta, E., Watanabe, H. \& Baba, T. All semiconductor low- $\Delta$ photonic crystal waveguide for semiconductor optical amplifier. Jpn. J. Appl. Phys. 45, 6116-6120 (2006).

84. Mingaleev, S. F., Miroshnichenko, A. E. \& Kivshar, Y. S. Low-threshold bistability of slow light in photonic-crystal waveguides. Opt. Express 15, 12380-12385 (2007).

85. Hirano, G. \& Koyama, F. Slowing light in Bragg reflector waveguide with tilt coupling scheme. Tech. Dig. IEEE/LEOS Annual Meet. 86-87 (Orlando, IEEE/LEOS, 2007).

86. Saleh, B.E.A., Teich, M.C. Fundamentals of Photonics 2nd Ed. (Wiley, New Jersey, 2007)

87. Dutton, C. Li, Z., Behroozi, C. H. \& Hau, L. V. Observation of coherent optical information storage in an atomic medium using halted light pulses. Nature 409, 490-493 (2001).

88. Julsgaard, B., Sherson, J., Cirac, J. I., Fiurásek, J., and Polzik, E. 
S. Experimental demonstration of quantum memory for light. Nature 432, 482-486 (2004).

89. Longdell, J. J., Fraval, E., Sellars, M. J. \& Manson, N. B. Stopped light with storage times greater than one second using electromagnetically induced transparency in a solid. Phys. Rev. Lett. 95, 063601 (2005).

90. Sakai, A., Hara G. \& Baba, T. Propagation characteristics of ultra-high $\Delta$ optical waveguide on silicon-on-insulator substrate. Jpn. J. Appl. Phys. 40, 383-385 (2001).

91. Silicon Photonics: An Introduction (eds Reed, G. T. \& Knight, A P.) (Wiley, New Jersey, 2004)

92. Optical Interconnects The Silicon Approach (eds Pavesi, L. \& Guillot, G.) (Springer, Berline, 2006).

93. Dulkeith, E., Xia, F., Schares, L., Green, W. M. J. \& Vlasov, Y. A. Group index and group velocity dispersion in silicon-on-insulator photonic wires. Opt. Express 14, 3853-3863 (2006).

94. Yamada, K., et al. Singlemode lightwave transmission in SOI-type photonic-crystal line-defect waveguides with phase-shifted holes. Electron. Lett. 38, 74-75 (2002).

95. Watanabe, Y., et al. Broadband waveguide intersection with low-crosstalk in two-dimensional photonic crystal circuits by sing topology optimization. Opt. Express 14, 9502-9507 (2006).

96. Ishii, S., Nozaki, K. \& Baba, T. Photonic molecules in photonic crystal. Jpn. J. Appl. Phys. 45, 6108-6111 (2006).

97. Nakagawa, A., Ishii, S. \& Baba, T. Photonic molecule lasers composed of GaInAsP microdisks. Appl. Phys. Lett. 86, 041112 (2005).

98. Ishii, S. \& Baba, T. Bistable lasing in twin microdisk photonic molecule. Appl. Phys. Lett. 87, 181102 (2005).

99. Astratov, V. N., Franchak, J. P. \& Ashili, S. P. Optical coupling and transport phenomena in chains of spherical dielectric microresonators with size disorder. Appl. Phys. Lett. 85, 5508-5510 (2004)

100. Möller, B. M., Woggon, U. \& Artemyev, M. V. Coupled-resonator optical waveguides doped with nanocrystals. Opt. Lett. 30, 2116-2118 (2005).

101. Hara, Y., Mukaiyama, T., Takeda, K. \& Kuwata-Gonokami, M. Heavy photon states in photonic chains of resonantly coupled cavities with supermonodispersive microspheres. Phys. Rev. Lett. 94, 203905 (2005)

102. Tanabe, T., et al. Fast all-optical switching using ion-implanted silicon photonic crystal nanocavities. Appl. Phys. Lett. 90, 031115 (2007)

103. Smith, D. D., Chang, H., Fuller, K. A., Rosenberger, A. T. \& Boyd R. W. Coupled-resonator-induced transparency. Phys. Rev. A 69, 063804 (2004)

104. Xu, Q., et al. Experimental realization of an on-chip all-optical analogue to electromagnetically induced transparency. Phys. Rev. Lett. 96, 123901 (2006).

105. Suematsu, Y. \& Furuya, K. Propagation mode and scattering loss of a two-dimensional dielectric waveguide with gradual distribution of refractive index. IEEE Trans. Microwave Theory Tech. MTT-20, 524-531 (1972)

106. Lee, K. K., Lim, D. R., Kimerling, L. C., Shin, J. \& Cerrina, F. Fabrication of ultralow-loss $\mathrm{Si} / \mathrm{SiO}_{2}$ waveguide by roughness reduction. Opt. Lett. 26, 1888-1890 (2001).

107. Yamada, K., et al. Microphotonics devices based on silicon wire waveguiding system. IEICE Trans. Electron. E87-C, 351-358 (2004).

108. Vlasov, Y. A. \& McNab, S. J. Losses in single-mode silicon-on-insulator strip waveguides and bends. Opt. Express 12, 1622-1631 (2004)

109. Tsang, H. K., et al. Optical dispersion, TPA and SPM in Si waveguides at $1.5 \mu \mathrm{m}$ wavelength. Appl. Phys. Lett. 80, 416-418 (2002)

110. Rieger, G. W., Virk, K. S. \& Young, J. F. Nonlinear propagation of ultrafast $1.5 \mu \mathrm{m}$ pulses in high-index contrast silicon-on-insulator waveguides. Appl. Phys. Lett. 84, 900-902 (2004).

111. Yamada, H., et al. Nonlinear-optic silicon-nanowire waveguides. Jpn. J. Appl. Phys. 44, 6541-6545 (2005).

112. Dulkeith, E., Vlasov, Y. A., Chen, X., Panoiu, N. C. \& Osgood Jr., R. M. Self-phase-modulation in submicron silicon-on-insulator photonic wires. Opt. Express 14, 5524-5534 (2006).

113. Oda, H., et al. Self-phase modulation in photonic-crystal-slab line-defect waveguides. Appl. Phys. Lett. 90, 231102 (2007).

114. Vlasov, Y. A. \& McNab, S. J. Coupling into the slow light mode in slab-type photonic crystal waveguides. Opt. Lett. 31, 50-52 (2006).

115. de Sterke, C. M., et al. Efficient slow light coupling into photonic crystals. Opt. Express 15, 10984-10990 (2007).

116. Ozaki, N., et al. High transmission recovery of slow light in a photonic crystal waveguide using a hetero group velocity waveguide. Opt. Express 15, 7974-7983 (2007).

117. Yang, L., et al. Topology optimisation of slow light coupling to photonic crystal waveguides. Electron. Lett. 43, 923-924 (2007).

118. Meier, M., et al. Laser action from two-dimensional distributed feedback in photonic crystals. Appl. Phys. Lett. 74, 7-9 (1999).

119. Sakoda, K., Ohtaka, K. \& Ueta, T. Low-threshold laser oscillation due to group-velocity anomaly peculiar to two- and three-dimensional photonic crystals. Opt. Express 4, 481-489 (1999).

120. Noda, S., Yokoyama, M., Imada, M., Chutinan, A. \& Mochizuki, M. Polarization mode control of two-dimensional photonic crystal laser by unit cell structure design. Science 293, 1123-1125 (2001).

121. Notomi, M., Suzuki, H. \& Tamamura, T. Directional lasing oscillation of two-dimensional organic photonic crystal lasers at several photonic band gaps. Appl. Phys. Lett. 78, 1325-1327 (2001).

122. Altug, H. \& Vuckovic, J. Experimental demonstration of the slow group velocity of light in two-dimensional coupled photonic crystal microcavity arrays. Appl. Phys. Lett. 86, 111102 (2005).

123. Sugitatsu, A. \& Noda, S. Room temperature operation of 2D photonic crystal slab defect-waveguide laser with optical pump. Electron. Lett. 39, 213-215 (2003).

124. Kiyota, K., Kise, T., Yokouchi, N., Ide, T. \& Baba, T. Various low group velocity effects in photonic crystal line defect waveguides and their demonstration by laser oscillation. Appl. Phys. Lett. 88, 201904 (2006).

125. Watanabe, H. \& Baba, T. High-efficiency photonic crystal microlaser integrated with a passive waveguide. Opt. Express $\mathbf{1 6}$ 2694-2698 (2008).

126. Clap, R., Dimitropoulos, D., Raghunathan, V., Han, Y. \& Jalali, B. Observation of stimulated Raman amplification in silicon waveguides. Opt. Express 11, 1731-1739 (2003).

127. Espinola, R. L., Dadap, J. I., Osgood, Jr., R. M., McNab, S. J. \& Vlasov, Y. A. Raman amplification in ultrasmall silicon-on-insulator wire waveguides. Opt. Express 12, 3713-3718 (2004).

128. Xu, Q., Almeida, R. \& Lipson, M. Demonstration of high Raman gain in a submicrometer-size silicon-on-insulator waveguide. Opt. Lett. 30, 35-37 (2005).

129. Espinola, R. J., Dadap, J. I., Osgood, Jr., R. M., McNab, S. J. \& Vlasov, Y. A. C-band wavelength conversion in silicon photonic wire waveguides. Opt. Express 13, $4341-4349$ (2005).

130. Fukuda, H., et al. Four-wave mixing in silicon wire waveguides. Opt. Express 13, 4629-4637 (2005).

131. Okawachi, Y., et al. All-optical slow-light on a photonic chip. Opt. Express 14, 2317-2322 (2006). 